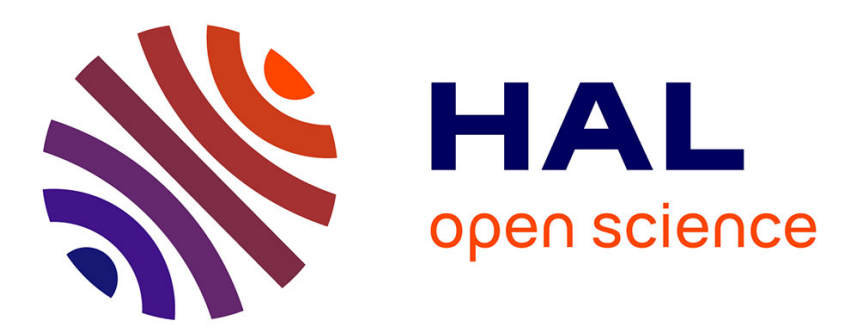

\title{
Mobile Measurements at 3.7 GHz Using a Massive MIMO Antenna Array in Outdoor Environments
} Nada Bel-Haj-Maati, Nadine Malhouroux-Gaffet, Patrice Pajusco, Michel Ney

\section{To cite this version:}

Nada Bel-Haj-Maati, Nadine Malhouroux-Gaffet, Patrice Pajusco, Michel Ney. Mobile Measurements at $3.7 \mathrm{GHz}$ Using a Massive MIMO Antenna Array in Outdoor Environments. EuCAP 2020: 14th European Conference on Antennas and Propagation, Mar 2020, Copenhague, Denmark. 10.23919/EuCAP48036.2020.9135314 . hal-02524303

\section{HAL Id: hal-02524303 https://hal.science/hal-02524303}

Submitted on 21 Feb 2022

HAL is a multi-disciplinary open access archive for the deposit and dissemination of scientific research documents, whether they are published or not. The documents may come from teaching and research institutions in France or abroad, or from public or private research centers.
L'archive ouverte pluridisciplinaire HAL, est destinée au dépôt et à la diffusion de documents scientifiques de niveau recherche, publiés ou non, émanant des établissements d'enseignement et de recherche français ou étrangers, des laboratoires publics ou privés. 


\title{
Mobile Measurements at $3.7 \mathrm{GHz}$ using a Massive MIMO antenna array in Outdoor Environments
}

\author{
Nada Bel-Haj-Maati ${ }^{12}$, Nadine Malhouroux ${ }^{1}$, Patrice Pajusco ${ }^{2}$, Michel $\mathrm{Ney}^{2}$ \\ ${ }^{1}$ Wireless and Propagation department, Orange Labs, Belfort, France \\ nada.belhajmaati@orange.com nadine.malhouroux@orange.com \\ ${ }^{2}$ Microwave department Lab-STICC, IMT Atlantique, Brest, France \\ patrice.pajusco@imt-atlantique.fr michel.ney@imt-atlantique.fr
}

\begin{abstract}
Massive multiple-input multiple-output (MIMO) is one of the investigated technologies for $5 \mathrm{G}$ deployment. This technology offers higher capacity, faster throughput and improved spectral and energy efficiency, thanks to the use of large-scale antenna arrays at the base station (BS), which enable beamforming and exploit the multipath richness. In this paper, we experimentally investigate massive MIMO propagation channel in several propagation environments. For this purpose, we have developed a new sounding experimentation using a large-scale planar array with about one thousand elements. Channel measurements were carried out at 3.7 GHz. We used a Virtual Planar Array (VPA) composed by an actual Uniform Linear Array (ULA) with 36 elements on the top of a moving vehicle to exploit its displacement to generate the second dimension of the VPA. A wideband channel sounder was used to record propagation channel transfer functions and to compute wideband characteristics. A new approach to estimate 3D direction of arrival (DoA) using a rectangular planar array is presented.
\end{abstract}

Index Terms-Massive MIMO, Channel Measurements, Propagation, Virtual Antenna Array, Outdoor environment, Delay spread, Angular spread, 5G, Base station, Antenna, Direction of arrival.

\section{INTRODUCTION}

Massive MIMO (multiple-input multiple-output) is a promising technology that can provide better services for the new generation of wireless communication (5G) [1] [2]. The key concept is to equip base stations with antenna arrays containing a large number of ports that enable beamforming. This allows many more users to transmit and receive data simultaneously over the same propagation channel. Thus, the development of advanced propagation model is required for advanced $5 \mathrm{G}$ deployment.

To characterize massive MIMO propagation channel, it is necessary to perform extensive measurement campaigns for statistical studies of spatio-temporal parameters in several environments. In this paper, wideband measurements were performed at $3.7 \mathrm{GHz}$ in mobile configuration using a wideband channel sounder and a virtual planar array (VPA). To this end, a uniform linear array (ULA) with 36-elements was mounted on the top of a vehicle and its move provided the second dimension of the array. Campus area and denseurban environment were investigated in both line-of-sight (LoS) and non-LoS (NLoS) conditions.
In this investigation, spatio-temporal characteristics: delay spread (DS), direction of arrival (DoA) and angular spread (AS), for both environments are evaluated and compared.

This paper is organized as follows. In Section II, the measurement setup is presented. Then in Section III, the outdoor measurement campaigns are described. Next, Section IV outlines measurement post processing analysis and describes the 3D-DoA estimation method. In Section V, results are presented for both environments. Finally, in Section VI, we summarize our contributions and draw conclusions.

\section{MEASUREMENTS SETUP}

\section{A. Channel sounder}

New sounder equipment has been developed to investigate the massive MIMO channel. This portable equipment, with SDR architecture, has been described in [3]. This sounder is based on pulse compression techniques with periodic signals. The multi-sensor measuring principle is based on a switching approach. This solution, usually adopted in channel sampling, reduces costs (a single receiver) and reduces calibration problems (a single active chain). Antenna switching control is performed in real time using the internal FPGA SDR receiver.

A GPS ensures the positioning and synchronization of all clocks, especially for MIMO measurements in an external context.

Finally, a PC ensures all the configurations and visualizations of the results. The assembly fully integrated in a $3 \mathrm{U}$ rack is shown in Fig. 1.

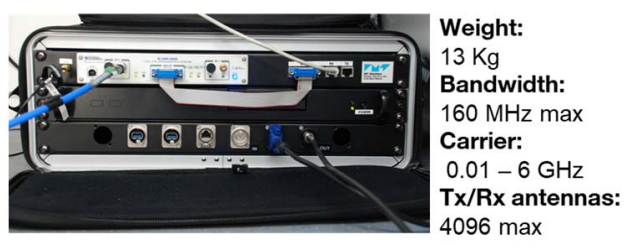

Fig. 1. Portable wideband channel sounder

\section{B. Antennas array}

A novel approach has been investigated to generate a flexible planar array. The $\mathrm{Rx}$ antenna was a ULA of 36 
isotropic monopole elements with a uniform spacing of $0.4 \lambda$ to avoid side-lobes, where $\lambda$ is the operating wavelength. The frequency band chosen is around $3.7 \mathrm{GHz}$, corresponding to a $5 \mathrm{G}$ frequency band.

The array was placed on a vehicle roof, as shown in Fig. 2, to synthesize a virtual planar array (Fig. 3) by obtaining the second dimension of the array through the displacement of the vehicle. The resulting planar array can contain more than a thousand of antennas. It ensures an excellent compromise between angular resolution (in both azimuth and elevation) and size array on the car.

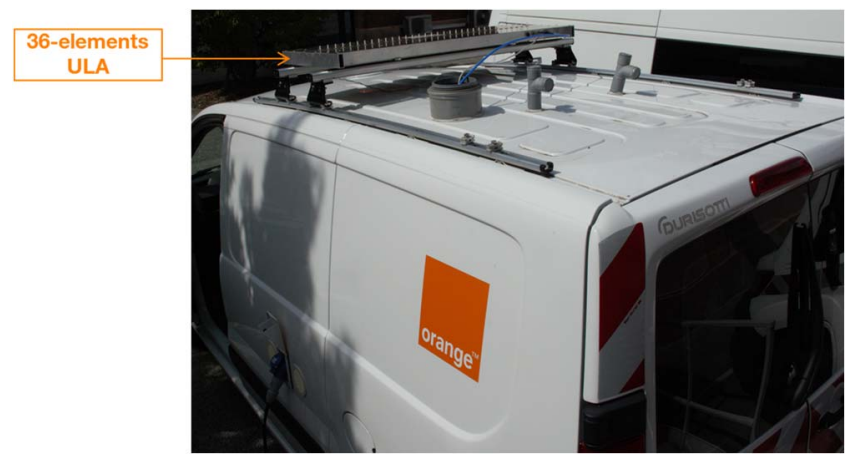

Fig. 2. 36-elements ULA mounted on the top of the vehicle

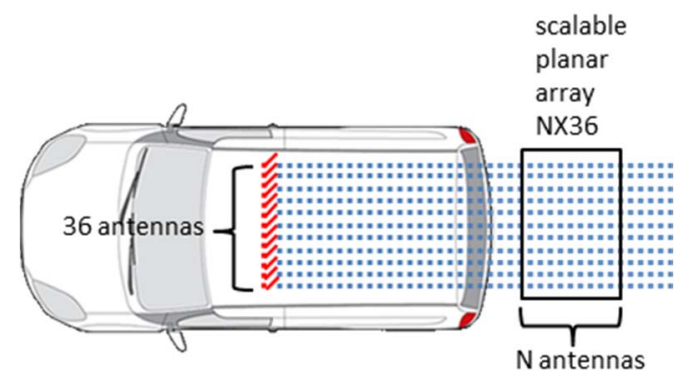

Fig. 3. Scalable planar array

At the transmitter side, a wideband omnidirectional antenna with vertical polarization was used. It was mounted on the roof on a measurements truck at different heights above floor level. Fig. 4 shows the Tx at 5 meters above ground.

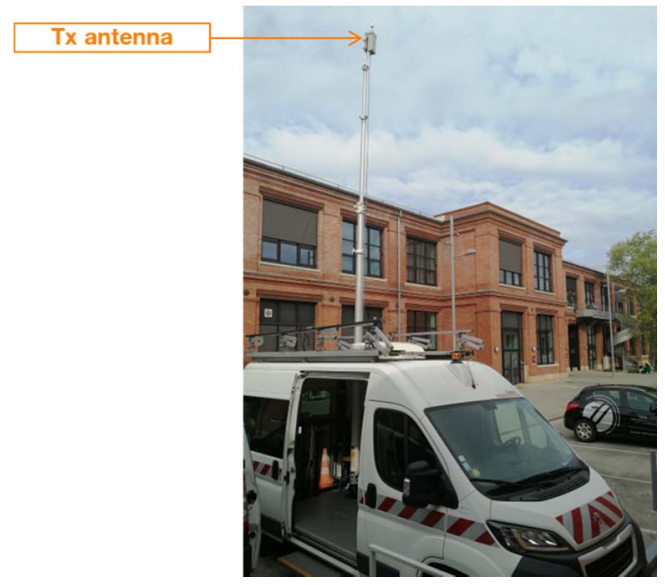

Fig. 4. Tx antenna on the roof of the measurements truck

\section{MEASUREMENT CAMPAIGNS}

The measurements were conducted in outdoor conditions, in two different environments: First, in a campus area (Techn'hom campus of Belfort), where the Orange Labs premises are located and in an urban area at Belfort city downtown.

The measured data were collected using the wideband channel sounder at $3.7 \mathrm{GHz}$, with an $80-\mathrm{MHz}$ channel bandwidth, and a sampling frequency of $100 \mathrm{MHz}$. The vehicle's speed was in the range of $20-30 \mathrm{~km} / \mathrm{h}$. The measurement settings are presented in Table I.

Table I. MEASUREMENT PARAMETERS

\begin{tabular}{|c|c|c|}
\hline Setup parameter & Suburban area & Urban area \\
\hline Carrier frequency $[\mathrm{GHz}]$ & \multicolumn{2}{|c|}{3.7} \\
\hline Bandwidth $[\mathrm{MHz}]$ & \multicolumn{2}{|c|}{80} \\
\hline Average Tx power $[\mathrm{dBm}]$ & \multicolumn{2}{|c|}{5} \\
\hline Length of transmit signal $[\mu \mathrm{s}]$ & \multicolumn{2}{|c|}{25} \\
\hline Average speed $[\mathrm{km} / \mathrm{h}]$ & $36 \times 105$ \\
\hline Rx antenna configuration & \multicolumn{2}{|c|}{2,16} \\
\hline ULA measurements time $[\mathrm{ms}]$ & \multicolumn{2}{|c|}{} \\
\hline
\end{tabular}

\section{A. Campus area}

The measurements were carried out in parking, in LoS configuration. The Tx antenna was placed at 5 and 10 meters above ground level. The Rx antenna was moving through different routes, as shown with different colours in Fig. 5.

Fig. 6 shows a picture taken during the measurement campaign.

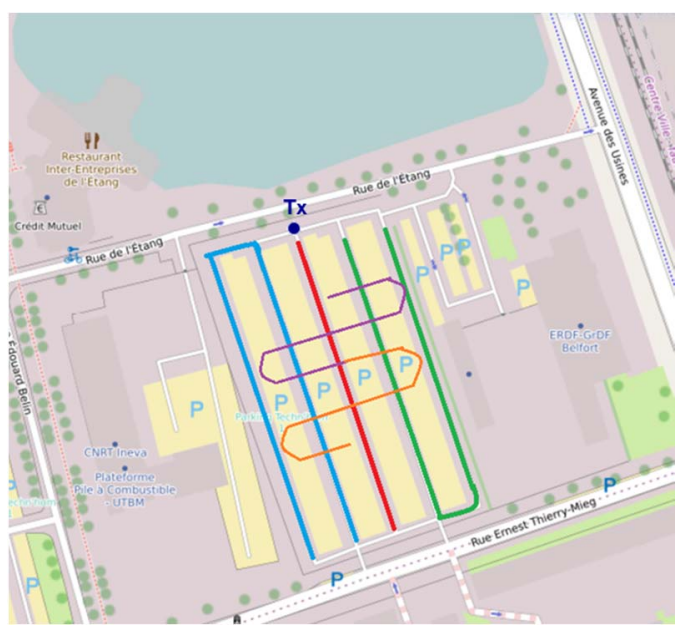

Fig. 5. Overview of measurements area with Tx position and $\mathrm{Rx}$ routes 


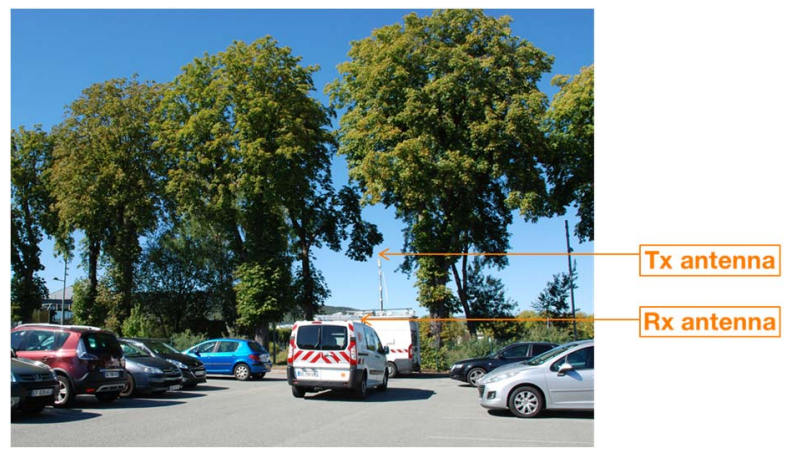

Fig. 6. Measurement environment with $\mathrm{Tx}$ and $\mathrm{Rx}$

\section{B. Urban area}

The measurement was performed in Belfort city downtown, in 3 different routes: one in LoS and 2 in NLoS configuration. The Tx antenna was placed at 10 meters above ground level, inside a street canyon with busy traffic. Fig. 7 represents the measurement area with Tx position and the 4 $\mathrm{Rx}$ routes.

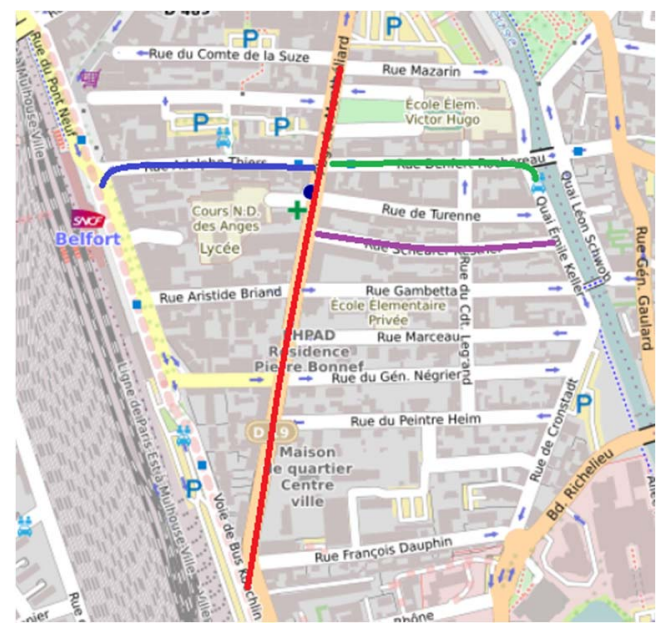

Fig. 7. Overview of urban environment with Tx and Rx routes

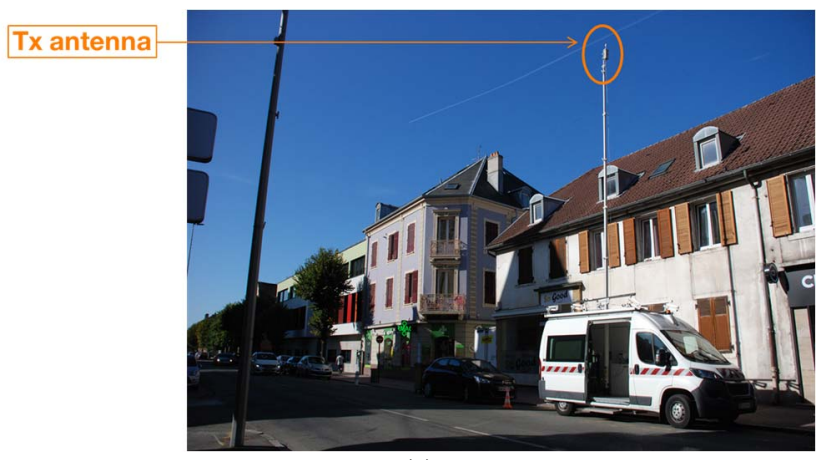

(a)

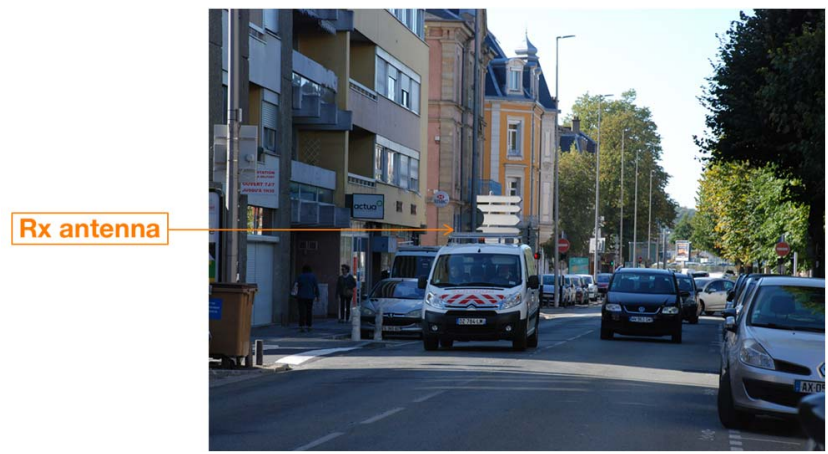

(b)

Fig. 8. Urban environement with: (a)- Tx and (b)- Rx

\section{DATA ANALYSIS}

The use of virtual array requires maintaining a constant speed during virtual array measurement. Each file represents a distance of a few meters. The traffic conditions prevent us to keep the same speed all the time. Moreover, there is a lack of sufficient dynamic in some collected impulse responses (IR). This is the reason why only IRs which had at least 30 $\mathrm{dB}$ dynamic range and a local constant speed has been considered. The IRs are collected using the hanning window to reduce the side lobes.

In this section, channel spatio-temporal parameters were extracted from measured IRs.

\section{A. Delay parameters}

The power delay profile (PDP) gives the distribution of signal power received over a multipath channel as a function of propagation delays. It is computed as the average power between the $N$ recorded IRs.

To characterize the multipath richness of a propagation channel, the rms delay spread (DS) is evaluated. The Rms DS is computed from PDP as (1):

$$
\tau_{\mathrm{DS}}=\sqrt{\frac{\int_{\tau}\left(\tau-\tau_{\text {mean }}\right)^{2} \operatorname{PDP}(\tau) \mathrm{d} \tau}{\int_{\tau} \operatorname{PDP}(\tau) \mathrm{d} \tau}}
$$

in which $\tau_{\text {mean }}=\frac{\int_{p} \tau \operatorname{PDP}(\tau) d \tau}{\int_{p} \operatorname{PDP}(\tau) d \tau}$ is the mean delay, the normalized first-order moment of the PDP.

\section{B. Angular parameters}

The spatial characteristics of the channel such as direction of arrival (DoA) and angular spreading, is important in channel modelling. Such information are required for localisation or MIMO communication studies. Several methods to estimate DoA are proposed in literature [7]. During measurements, a preview of the DoA is obtained via a double FFT. For post processing, a 3D-DoA novel approach based on parametric modeling of measured data (IR) is used. It is a more accurate method, easy to apply in engineering practice, and reduced computational complexity. This provide also a discrete and compact représentation of channel measurements. 
We consider a receiving rectangular UPA consisting of $\mathrm{N}$ ULA with $M$ antenna ports in each array. The spacing between two ULA depends on the vehicle speed $\mathrm{v}$ and the measurements time $\mathrm{t}_{\text {meas }}\left(\Delta \mathrm{x}=\mathrm{v} \times \mathrm{t}_{\text {meas }}\right)$, and it is fixed $(\Delta y=0.4 \lambda)$ in $y$-axis. The measured data can be denoted as:

$$
h(\tau, x, y)=\sum_{k} A_{k}(\tau, x, y) e^{-i 2 \pi\left(f_{x_{k}} x+f_{y_{k}} y\right)}
$$

where $\left\{\begin{array}{l}\mathrm{f}_{\mathrm{x}_{\mathrm{k}}}=\cos \left(\phi_{\mathrm{k}}\right) \sin \left(\theta_{\mathrm{k}}\right) \\ \mathrm{f}_{\mathrm{y}_{\mathrm{k}}}=\sin \left(\phi_{\mathrm{k}}\right) \sin \left(\theta_{\mathrm{k}}\right)\end{array}\right.$, with $\theta_{\mathrm{k}}$ is the elevation angle and $\phi_{k}$ the azimuth angle of the $k^{\text {th }}$ ray path, and $A_{k}(\tau, x, y)$ its amplitude.

According to equation (3), the estimation of the DoA $(\theta$ and $\phi)$ is denoted by estimating the data parameters $f_{x}$ and $\mathrm{f}_{\mathrm{y}}$. To that end, we use an iterative algorithm such as Clean method [4], Fig. 9 gives the major algorithmic steps.

$$
\left\{\begin{array}{c}
\phi=\arctan \left(\frac{f_{y}}{f_{x}}\right) \\
\theta=\sin ^{-1}\left(\sqrt{f_{x}^{2}+f_{y}^{2}}\right)
\end{array}\right.
$$

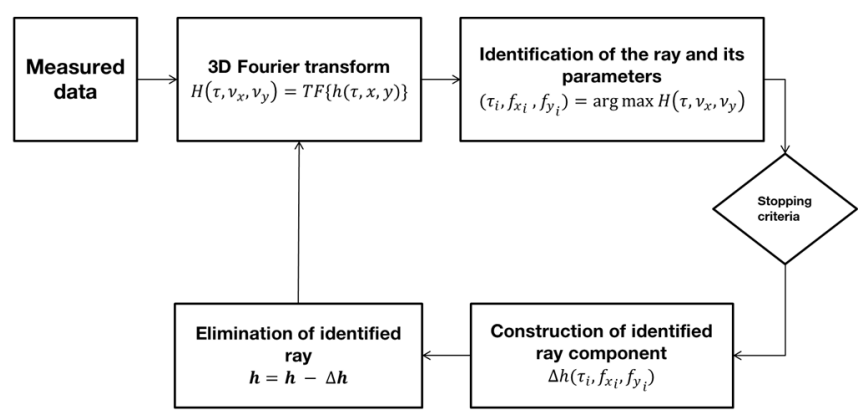

Fig. 9. Algorithmic steps of 3D-DoA estimation

To characterize the angular dispersion of propagation channels, angular spread (AS) was evaluated. Indeed, different definitions exist in the literature, such as Fleury [5] and 3GPP [6] methods. In this paper, the 3GPP method which is the most commonly applied is used:

$$
\begin{gathered}
\sigma_{\theta}=\min _{\Delta} \sigma_{\theta}(\Delta) \\
\text { with: } \sigma_{\theta}(\Delta)=\sqrt{\frac{\sum_{\mathrm{n}}\left(\theta_{\mathrm{n}}+\Delta-\bar{\theta}\right)^{2} \mathrm{P}_{\mathrm{n}}}{\sum_{\mathrm{n}} \mathrm{P}_{\mathrm{n}}}}, \quad \bar{\theta}=\frac{\sum_{\mathrm{n}}\left(\theta_{\mathrm{n}}+\Delta\right) \mathrm{P}_{\mathrm{n}}}{\sum_{\mathrm{n}} \mathrm{P}_{\mathrm{n}}}
\end{gathered}
$$

where $P_{n}$ is the power and $\theta_{n}$ the DoA of the nth rays, and $\Delta$ is a linear shift angle

\section{RESULTS ANALYSIS}

In this section, results are presented for which TX is at $10 \mathrm{~m}$ from the ground level. For each environment, PDP and DoA are depicted and the RMS delay spreads were determined for $20 \mathrm{~dB}$ of dynamic.

For both urban and campus environments, we present first an example of a route in LoS conditions for campus and in LoS and NLoS conditions for urban (Fig. 10). Two Rx positions were studied for each case: R1 and R2 (campus), and Rlos and Rnlos (urban). Corresponding PDPs are depicted in Fig. 11 and Fig.12, respectively. The 3D-DoA are also computed using the method described in the previous section for validation. Results are shown in Fig. 13.

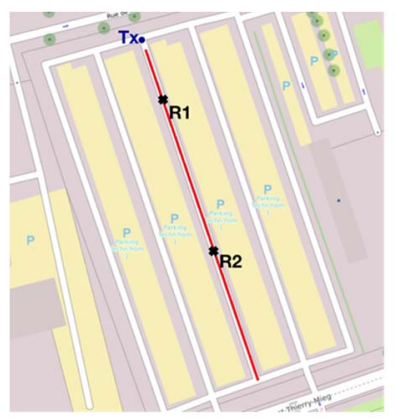

Campus

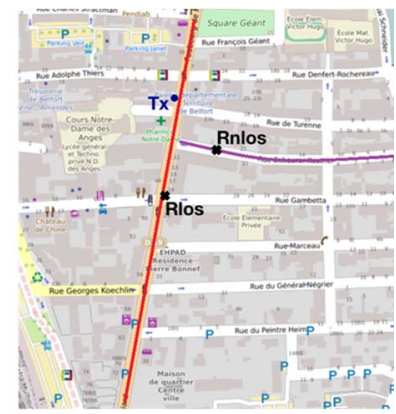

Urban
Fig. 10. Example of route in LoS with two Rx positions

\section{A. Campus environment}

The multipath richness can be observed in both R1 and R2 PDPs. This is due to the numerous cars parked on the site, and also to the metallic building near the measurement area. The studied route contains $50 \mathrm{Rx}$ positions ranged from 5 to $103 \mathrm{~m}$, which DS values that vary from 11 to 87 ns.

As shown in Fig. 13, it is possible to identify the main DoA for both $\mathrm{Rx}$ positions which corresponds to the direct ray path. For R2 situated at $63 \mathrm{~m}$ from Tx, an additionnal ray is found. It may be due to a ground reflexion.

\section{B. Urban environment}

The PDP for Rnlos position in NLoS conditions consists of several rays with close delays. The studied route in LoS contains $124 \mathrm{Rx}$ positions ranged from 20 to $130 \mathrm{~m}$, and the one in NLoS $40 \mathrm{Rx}$ positions ranged from 49 to $83 \mathrm{~m}$. The delay spread in NLoS (39 ns) is larger than the one in LoS (18 ns) due to multipath.

From the computed 3D-DoA (Fig. 13), we observe that the DoA with highest power corresponds to direct ray path for Rlos. Another ray path is identified with the same azimuth angle but with less elevation. It can correspond to a reflexion on a top of a vehicle. Two directions of arrival are found for Rnlos position. The strongest can be a diffraction effect and the second one may be due to a ground reflexion.
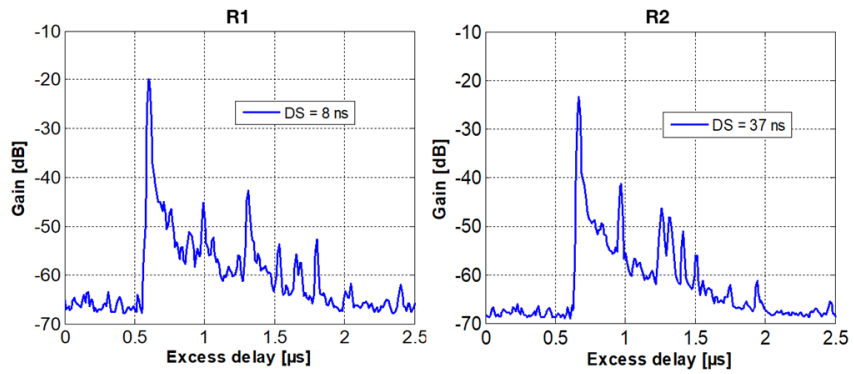

Fig. 11. PDPs for R1 and R2 positions 

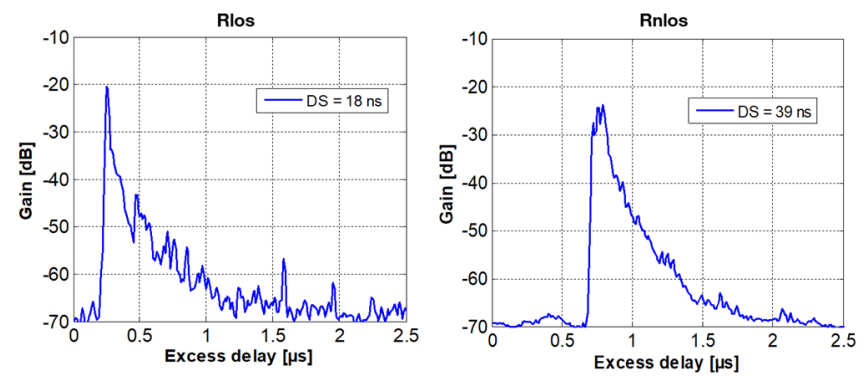

Fig. 12. PDP for Rlos and Rnlos positions
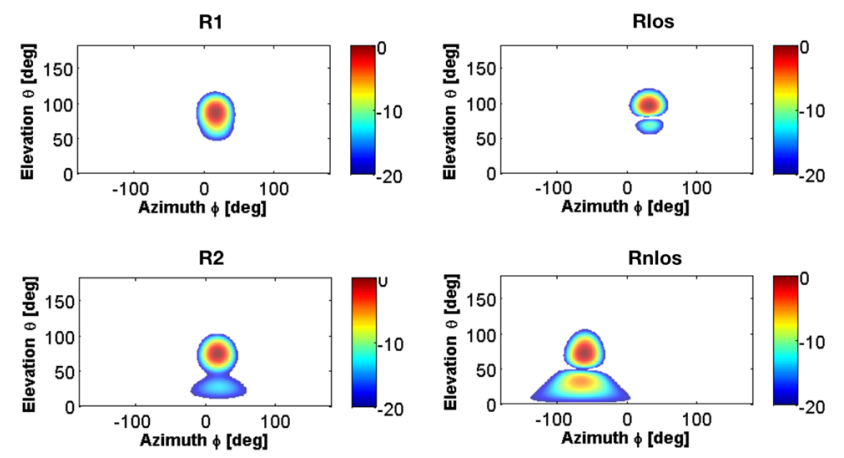

Fig. 13. DoA computed for R1, R2, Rnlos and Rlos

\section{Comparison}

In this section we compare the massive MIMO propagation channel performances in campus and urban environments. The spatio-temporal parameters DS and AS distributions are investigated considering around 130 to 170 collected data (Fig. 14 and Fig. 15).

The DS values are practically constant in LoS urban environment, while it varies significantly in both NLoS (40 to $90 \mathrm{~ns}$ ) and campus environment (10 to $90 \mathrm{~ns}$ ). This is due to the multipath effect caused by cars and buildings. DS values are in agreement with literature [8].

The azimuth AS distribution (Fig. 15) is more important in both campus and NLoS urban environments $\left(10^{\circ}\right.$ to $\left.35^{\circ}\right)$, instead of $\left(10^{\circ}\right.$ to $\left.16^{\circ}\right)$ in urban LoS. The elevation AS is almost constant $\left(\sim 9^{\circ}\right)$ for LoS cases in both environments, varies from $8^{\circ}$ to $14^{\circ}$ for NLoS case.

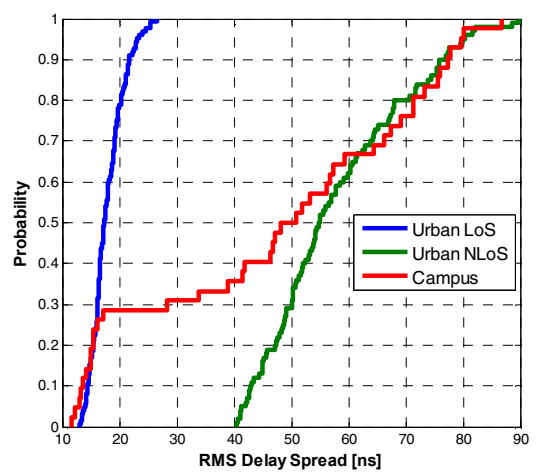

Fig. 14. RMS delay spread distribution
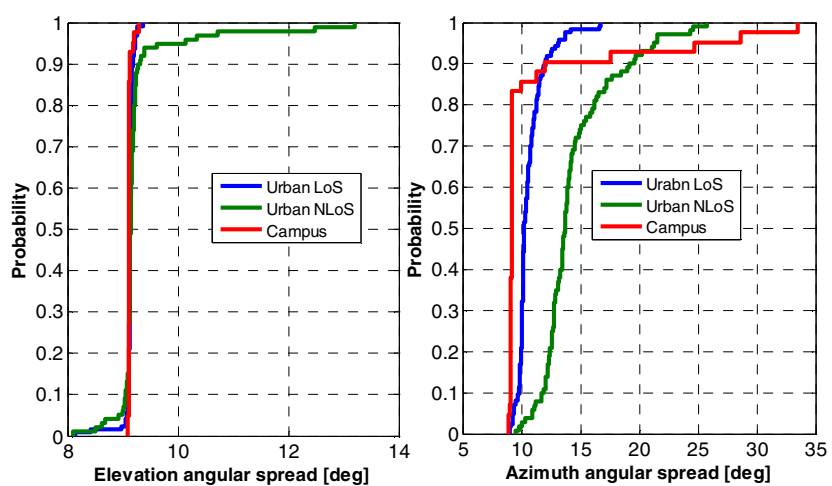

Fig. 15. Angular spread distribution in elevation and azimuth

\section{CONCLUSION}

In this paper, mobile measurements using a massive MIMO antenna array were carried out in a typical 5G context: at $3.7 \mathrm{GHz}$ in two outdoor environments (campus and urban in Belfort city downtown). A novel 3D-DoA estimation approach is presented. It was applied to the real collected data to compute 3D-DoA to validate our method. Delay and angular spread have been compared for both environments. Delay spread values are less than $90 \mathrm{~ns}$ for both environments and their average is less than $60 \mathrm{~ns}$. Angular spread values do not exceed $35^{\circ}$ in azimuth and $14^{\circ}$ in elevation. Moreover, the dispersion in azimuth is much larger than in elevation. Further work will study other environments (rural, suburban, dense urban).

\section{ACKNOWLEDGMENT}

This study has been completed under a research contract between Orange and IMT Atlantique.

We thank P. Brun, C. Moroni, J.M. Conrat for their help and skills during the measurement campaigns in Belfort.

\section{REFERENCES}

[1] E. Larsson, O. Edfors, F. Tufvesson, T. Marzetta," "Massive MIMO for next generation wireless systems," IEEE Communications Magazine, pp. 186-195, vol52, no.2, Feb. 2014.

[2] H. Q. Ngo, E. Larsson, and T. Marzetta, "Energy and spectral efficiency of very large multiuser MIMO systems," IEEE Transaction on Communications, vol. 61, no.4, p.1436-1449,2013.

[3] P. Pajusco, E. Bel Kamel, N. Malhouroux and R. Masood, "Space time channel sounding method using SAR approach," in $201913^{\text {th }}$ European Conference on Antennas and Propagation (EUCAP), 2019.

[4] A. Segalovitz, B. Roy Frieden, "A CLEAN-type deconvolution algorithm", Astronomy \& Astrophsics, vol. 70, no. 3, pp. 335-343, 1978.

[5] Fleury. B, "First- and second-order characterization of direction dispersion and space selectivity in the radio channel," Information theory, IEEE Transactions on 46(6), 2027-2044.

[6] 3GPP-3GPP2 SCM AHG, "SCM-135: Spatial channel model text description", August 2003.

[7] R. Muhamed, "Direction of arrival estimation using antenna arrays", Thesis, Virginia Tech, 1996..

[8] V. Kristem, C. U. Bas, R. Wang, and A.F. Molisch, "Outdoor Wideband Channel Measurements and Modeling in the $3-18 \mathrm{GHz}$ Band," IEEE Transactions on Wireless Communications, vol. 17, pp. 4620-4633, 2018. 